\title{
Atomically resolved structure of InAs quantum dots
}

Cite as: Appl. Phys. Lett. 78, 2309 (2001); https://doi.org/10.1063/1.1365101

Submitted: 21 November 2000 . Accepted: 16 February 2001 . Published Online: 10 April 2001

J. Márquez, L. Geelhaar, and K. Jacobi

\section{ARTICLES YOU MAY BE INTERESTED IN}

Erratum: "Atomically resolved structure of InAs quantum dots" [Appl. Phys. Lett. 78, 2309 (2001)]

Applied Physics Letters 90, 129902 (2007); https://doi.org/10.1063/1.2715039

Direct formation of quantum-sized dots from uniform coherent islands of InGaAs on GaAs surfaces

Applied Physics Letters 63, 3203 (1993); https://doi.org/10.1063/1.110199

Shape transition of InAs quantum dots by growth at high temperature

Applied Physics Letters 74, 1224 (1999); https://doi.org/10.1063/1.123506
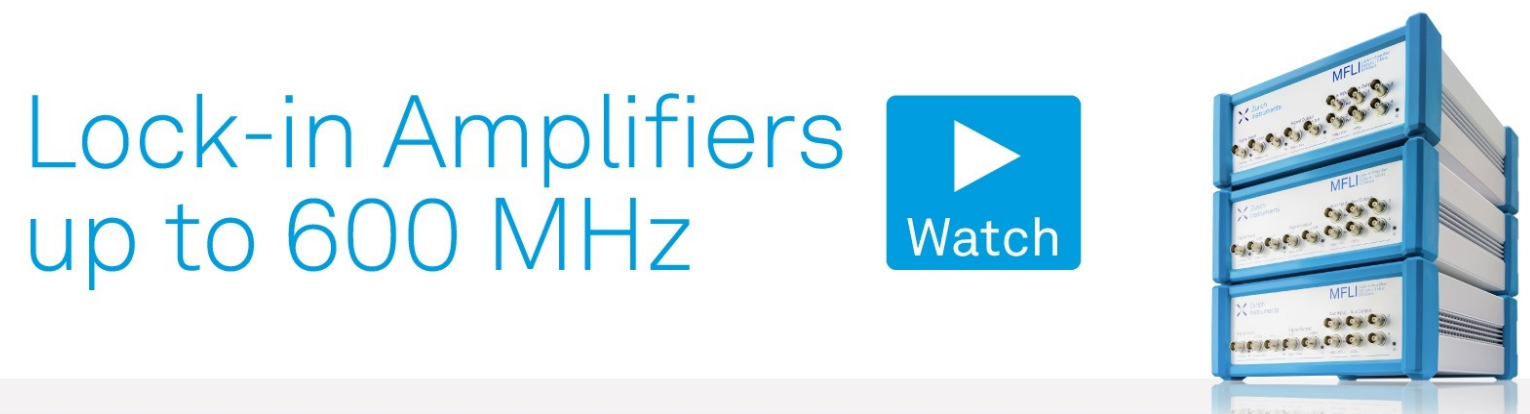

Appl. Phys. Lett. 78, 2309 (2001); https://doi.org/10.1063/1.1365101

78, 2309

(C) 2001 American Institute of Physics. 


\title{
Atomically resolved structure of InAs quantum dots
}

\author{
J. Márquez, L. Geelhaar, and K. Jacobia) \\ Fritz-Haber-Institut der Max-Planck-Gesellschaft, Faradayweg 4-6, D-14195 Berlin, Germany
}

(Received 21 November 2000; accepted for publication 16 February 2001)

\begin{abstract}
InAs was grown by molecular-beam epitaxy onto $\mathrm{GaAs}(001)$ until quantum dots (QDs) formed. At this point, the growth was interrupted and the uncovered QDs were investigated in situ by scanning tunneling microscopy (STM). Atomically resolved STM images of the QDs revealed that four dominating bounding facets occur, whose Miller indices were identified to be $\{137\}$. The assignment of the facet orientation was based on experiments on planar high Miller index GaAs surfaces. In addition, the latter experiments indicated that $\{137\}$ facets are thermodynamically stable only up to a certain size. This conclusion is assumed to explain the sharp size distribution of InAs QDs.

(c) 2001 American Institute of Physics. [DOI: 10.1063/1.1365101]
\end{abstract}

In recent years, the self-organized growth of semiconductor nanostructures that show quantum-size effects has been of considerable interest due to their enormous potential for technological applications. ${ }^{1}$ Consequently, laser devices operating with self-assembled InAs quantum dots (QDs) embedded in GaAs have already been demonstrated. ${ }^{2}$ So far, experimental work has focused on varying the growth conditions to control the size and improve the uniformity of the QDs. ${ }^{3}$ Theoretical work has addressed the formation phenomena of quantum dots and attempted to predict their size and density. ${ }^{4}$ However, the accuracy of such calculations is limited by the knowledge on the QD shape. While most theoretical calculations have assumed that only surfaces with low Miller indices occur as bounding facets of the QDs, particularly those which are based on thermodynamical descriptions, ${ }^{4}$ from experiments the occurrence of facets with high Miller indices was concluded. ${ }^{5-9}$ Thus, the determination of the exact shape of the QDs is needed to substantiate the theoretical models and thereby, to improve the device technology.

In this letter, we determine the exact shape of selfassembled InAs QDs from atomically resolved structures on their bounding facets. Four facets dominate the shape of the QDs, whose Miller indices were identified with the help of studies on high-index GaAs surfaces to be $\{137\}$.

The experiments were performed in a multichamber ultra-high-vacuum system. ${ }^{10}$ Our samples, with a typical size of $10 \times 10 \mathrm{~mm}^{2}$, were cut from GaAs(001) epiready wafers [Wafer Technology, Si doped, $n=(1.1-4.8) \times 10^{18} \mathrm{~cm}^{-3}$ ]. GaAs buffer layers of $20-50 \mathrm{~nm}$ thickness were grown by molecular-beam epitaxy at a temperature of $560^{\circ} \mathrm{C}$. After growth, the samples were cooled down to $450{ }^{\circ} \mathrm{C}$ and were kept at this temperature for 10 min until the $\mathrm{GaAs}(001) c(4$ $\times 4)$-diffraction pattern was observed by reflection highenergy electron diffraction (RHEED). Hereafter, $1.8 \pm 0.2 \mathrm{ML}$ InAs were deposited at a growth rate of $0.05 \AA \mathrm{s}^{-1}$. The As:In beam equivalent pressure was 40. RHEED showed a transition from a streaky to a spotty pattern, indicating the onset of three-dimensional islanding. The samples were then

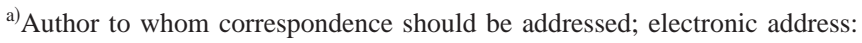
jacobi@fhi-berlin.mpg.de
}

rapidly (within $15 \mathrm{~s}$ ) transferred to the analysis chamber where they cooled down to room temperature before they were examined by scanning tunneling microscopy (STM). STM images were acquired in constant-current mode.

As a first observation, the RHEED pattern (not shown here) showed the often observed chevron-like spots along the [1 $1 \overline{1} 0]$ azimuth, which is attributed to facets that are inclined at an angle of $20^{\circ}-25^{\circ}$ from the substrate. Perpendicularly, i.e., along [110], the RHEED pattern remained spotty, indicating that no specific facet orientation occurred. Furthermore, large scale $\left(0.5 \times 0.5 \mu \mathrm{m}^{2}\right)$ STM images (not shown here) revealed a QD density of $1.9 \times 10^{11} \mathrm{~cm}^{-2}$ with a very sharp size distribution. The average diameter and height of the QDs were 125 and $22 \AA$, respectively. Since both RHEED and STM results were in good accord with observations of other studies (e.g., Refs. 5 and 9), we conclude that our further results should hold in general for InAs QDs. However, it should be noted that our study is based on one specific set of growth parameters, while it is well known that the size, the composition, and the density of the QDs depend on the growth conditions. ${ }^{3,11}$ Therefore, our analysis does not exclude that QDs of different shapes may also occur.

In order to determine the shape of the QDs, STM images showing smaller areas $\left(<800 \times 800 \AA^{2}\right)$ were acquired. With the help of the atomic structures of the wetting layer, which exists between the QDs, we have been able to calibrate the scanner and to determine the principal directions

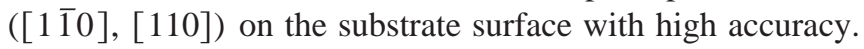

Figure 1(a) shows a STM image of one of the QDs, which is representative for all QDs considered for this letter. The QD has a pyramidal shape with a fairly sharp summit. There are four pronounced facets at the right- and left-hand sides of the image, which determine the shape of the QD. Most remarkably, atomic features are clearly visible as rows of humps on these four facets. The inclination angle between these rows and the substrate is $26^{\circ} \pm 2^{\circ}$. The intersections of these facets with the substrate are not parallel to the principal axes of the (001) substrate, but are inclined by $24^{\circ} \pm 2^{\circ}$ with respect to $[\overline{1} 10]$. We note that the quantity of the error was derived from a total amount of 53 QDs. The ends of the QD on the lower and upper parts of the image do not show clear facets and appear fairly rounded. Atomic-scale features were 

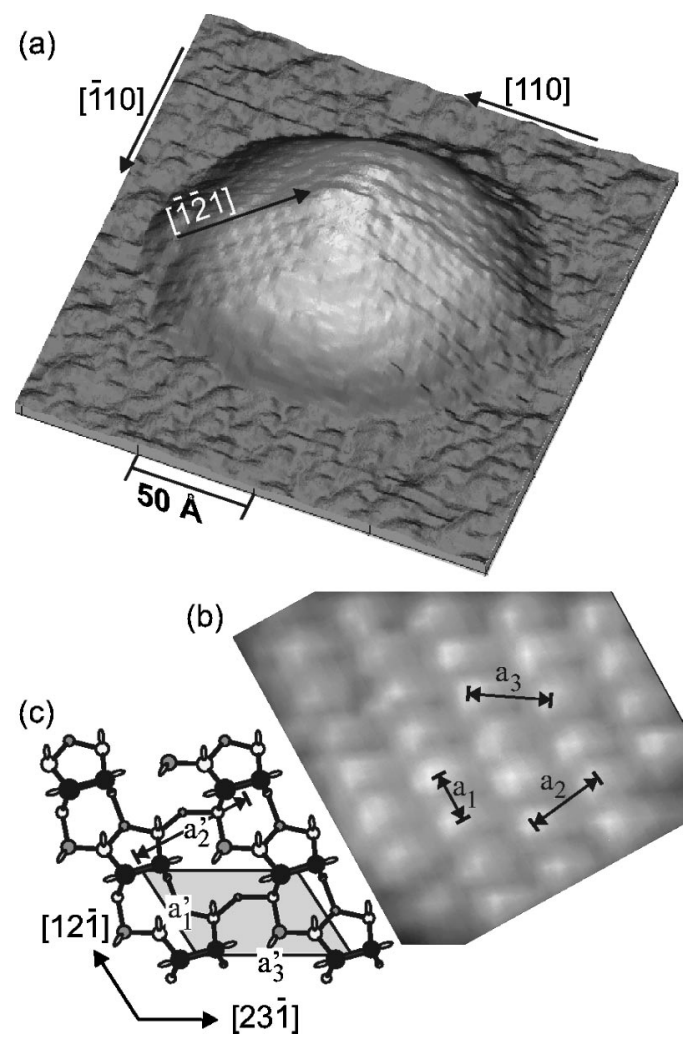

FIG. 1. (a) Three-dimensional STM image of an uncovered InAs QD grown on $\operatorname{GaAs}(001)$. (b) Zoom on one of the $\{137\}$ oriented facets of the QD. $U$ $=-2.75 \mathrm{~V}, I=0.15 \mathrm{nA}$. (c) Ball and stick model for the reconstructed InAs(137) surface. The gray rectangle shows the unit cell. As atoms are represented by gray, In atoms by white circles. As dimers are highlighted by black circles.

not resolved in these QD parts on any STM image. Despite the exceptional spatial resolution on the facets, the determination of the Miller indices of these facets based on all angles is a difficult task, since the error in the measurements is still too large.

Instead, to determine the Miller indices of the four specific facets we examine the atomic features within the facets of the QDs in more detail. In order to visualize these atomic features Fig. 1(b) shows an image of one of these facets, where a linear slope is subtracted to have equal contrast throughout the image. There are rows of humps running from the bottom to the top of the image. Since the image was acquired with negative sample voltage, i.e., filled states were imaged, it is reasonable to assign the humps to As-derived states. The characteristic distances, labeled in the image $a_{1}$, $a_{2}$, and $a_{3}$ between the humps are $6.5 \pm 0.1,9.0 \pm 0.3$, and $10.5 \pm 0.2 \AA$, respectively. The connection lines parallel to $a_{1}$ and $a_{2}$ meet at an angle of $82.4^{\circ} \pm 0.7^{\circ}$. The determination of the Miller indices was achieved by comparing the arrangement of the humps on the facet with the arrangement of As atoms of known GaAs surfaces. A perfect agreement was found in a comparison with a surface which was discovered by an investigation of surfaces inside the stereographic triangle. ${ }^{12}$ In those experiments a new stable surface was found, whose orientation lies within the range of the angles of the QD facets. The Miller indices of this surface were identified in further experiments to be $(2511) .^{13}$

The GaAs(2511) surface consists of a series of three As dimers aligned along [12ī], which are tilted with respect to

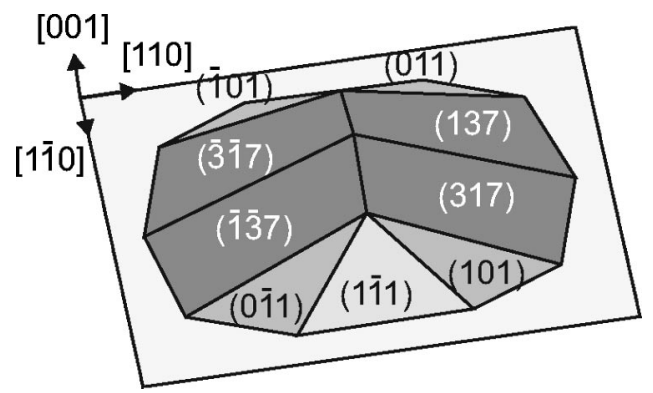

FIG. 2. Extracted shape of an InAs QD. The $\{101\}$ and $\{111\}$ surfaces were not determined with high accuracy and are only included to complete the model.

the $(2511)$ surface plane. Adjacent series of three As dimers are slightly shifted towards [23ī] and give the surface a striped appearance. The orientation of these stripes is (137). The arrangement of the As dimers within the (137) stripes is shown in a ball and stick model in Fig. 1(c). The connection between $\operatorname{GaAs}(2511)$ and the QDs is that the arrangement of As dimers on the (137) stripes is the same as that of the humps on the QD facets. For comparison, the corresponding distances of the As dimers on the (137) stripes are 6.9, 8.9, and $10.6 \AA$, for $a_{1}^{\prime}, a_{2}^{\prime}$, and $a_{3}^{\prime}$, respectively. The angle between $a_{1}^{\prime}$ and $a_{2}^{\prime}$ is $82.6^{\circ}$. The accuracy of our determination of the QD shape comes from the fact that distances of atomic features were considered, which originate from the unit mesh of a reconstruction. Hence, they can only amount to discrete values, which are much larger than the error of the measurement. Thus, the experimental results show unambiguously that the QD facets have the orientation (137).

Based on these results, we propose a model for the shape of self-assembled InAs QDs, which is depicted in Fig. 2. The InAs QDs are bounded by at least ten facets. Four of them determine the shape and are of $\{137\}$ orientation. The other facets are not well resolved and do not show clear edges. Thus, it is questionable to assign specific orientations to them. Nearby planes are $\{10 n\}$ and $\{11 \bar{n}\} \mathrm{B},(n=1,2,3)$.

This shape of the QDs may account for the numerous models and experimental results reported so far. ${ }^{5-9}$ Figure 3 shows height profiles of a typical QD along (a) [1 $\overline{1} 0]$ and (b) [110]. The right-hand sides of Figs. 3(a) and 3(b) present typical three-dimensional (3D) gray-scale images of the QDs. A shape anisotropy along the two axes is clearly visible. The height profile along [1 $1 \overline{1} 0]$ is rounded, whereas the profile in the perpendicular direction is triangular. It is clear

(a) $\AA$
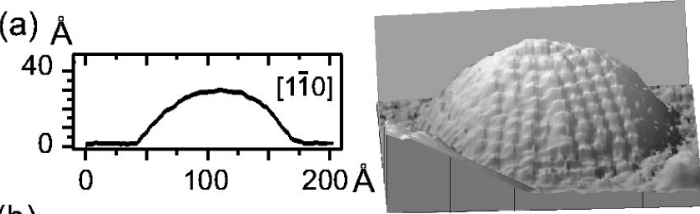

(b)
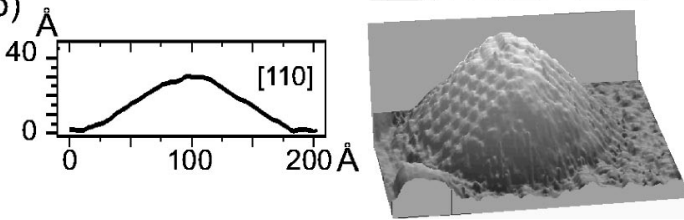

FIG. 3. Height profiles of a QD, parallel to the (a) [1 10$]$ and (b) [110] directions. The images on the right-hand side show the respective 3D STM image of the QD. $U=-2.75 \mathrm{~V}, I=0.15 \mathrm{nA}$. 
that different shapes of the QDs would be derived if only one of these cross sections was considered: From the first one a lens-shaped QD would be derived, and a pyramidal QD in the second case. Furthermore, this anisotropy explains the difference in the RHEED patterns taken along these directions. With the electron beam directed along [110], the RHEED pattern shows only a spotty pattern. Since the cross section of the QD along [1 $\overline{1} 0]$ is rounded, the intensity of the diffracted electron beams is distributed homogeneously, and thereby no facet-related spots can be observed. In contrast, parallel to $[1 \overline{1} 0]$ the electrons are diffracted from the $\{137\}$ facets of the QDs towards specific directions, and thereby the chevrons appear in the RHEED pattern. Moreover, the agreement between RHEED and STM observations also ensures that the shape of the QDs is determined during growth and is not related to the quench procedure of the samples. This conclusion is also in accord with experiments by Guha, Madhukar, and Rajkumar, ${ }^{14}$ who reported a similar cross-sectional anisotropy on buried InAs QDs by highresolution transmission electron microscopy.

The occurrence of $\{137\}$ facets on the QD leads to significant conclusions for the formation of InAs QDs. The study of surfaces inside the stereographic triangle ${ }^{12}$ revealed that only one thermodynamically stable surface exists within the observed region, namely, the $\operatorname{GaAs}(2511)$ surface. This surface is composed of three As dimers wide (137) stripes, which by themselves do not fulfill the electron-counting rule (ECR).$^{15}$ The ECR states that the reconstructed surface remains semiconducting, and thereby it is expected to be of low energy. In contrast, the GaAs(2 511$)$ surface does fulfill the ECR, because threefold coordinated $\mathrm{Ga}$ atoms within the trenches between adjacent (137) stripes exist, which compensate the missing charges of the surface yielding a semiconducting surface reconstruction. ${ }^{13}$ However, as demonstrated in Refs. 12 and 13, and (137) stripes with different widths also occur. These stripes extend over several $100 \AA$ and indicate that a surface, which is composed of (137) stripes with other than three As dimers should be at least semistable. In fact, theoretical studies in Ref. 13 have shown that the surface energy of one of these surfaces, which did not fulfill the ECR either, was only a little higher than that of the GaAs(2 511) surface. Thus, the experimental observations on $\operatorname{GaAs}(2511)$ and the theoretical calculations led us to the assumption that $\{137\}$ areas are stable up to a certain size. Above this size, the surface energy per unit area increases rapidly. This may explain the sharp size distribution of the QDs with a modified coherent Stranski-Krastanow growth mode: When the area of the $\{137\}$ facets on the growing QD reaches this certain size, the energy gain due to the strain relaxation within the three-dimensional island does no longer exceed the surface energy. Hence, a certain critical QD size exists, which is determined by the size of the $\{137\}$ facets.

To summarize, based on atomically resolved STM images of the QD facets, we proposed a model for the shape of self-assembled InAs QDs, which is determined by four $\{137\}$ facets. The ends of the QDs in the [1T̄0] direction are rounded and do not show specific facets. It is suggested that the special arrangement of As dimers within the $\{137\}$ orientations and the resulting surface energies might be responsible for the sharp size distribution of self-assembled InAs QDs and should, therefore, be considered in further theoretical studies.

The authors would like to thank P. Kratzer for fruitful discussions and P. Geng for technical assistance. This work was supported by the Deutsche Forschungsgemeinschaft (Sonderforschungsbereich 296, Project A2) and by the German Bundesministerium für Bildung und Forschung under Grant No. 05622 EBA4.

${ }^{1}$ D. Bimberg, M. Grundmann, and N. N. Ledentsov, Quantum Dot Heterostructures (Wiley, Chichester, NY, 1999).

${ }^{2}$ See, e.g., M. Grundmann, Physica E (Amsterdam) 5, 167 (2000), and references therein.

${ }^{3}$ G. S. Solomon, J. A. Trezza, A. F. Marshall, and J. S. Harris, Jr., Phys. Rev. Lett. 76, 952 (1996); I. Mukhametzhanov, R. Heitz, J. Zeng, P. Chen, and A. Madhukar, Appl. Phys. Lett. 73, 1841 (1998); H. Saito, K. Nishi, and S. Sugou, ibid. 74, 1224 (1999); Z. R. Wasilewski, S. Fafard, and J. P. McCaffrey, J. Cryst. Growth 201/202, 1131 (1999).

${ }^{4}$ See, e.g., N. Moll, M. Scheffler, and E. Pehlke, Phys. Rev. B 58, 4566 (1998); L. G. Wang, P. Kratzer, N. Moll, and M. Scheffler, ibid. 62, 1897 (2000), and references therein.

${ }^{5}$ Y. Nabetani, T. Ishikawa, S. Noda, and A. Sasaki, J. Appl. Phys. 76, 347 (1994).

${ }^{6}$ J. M. Moison, F. Houzay, F. Barthe, L. Leprince, E. Andráe, and O. Vatel, Appl. Phys. Lett. 64, 196 (1994)

${ }^{7}$ Y. Hasegawa, H. Kiyama, Q. K. Xue, and T. Sakurai, Appl. Phys. Lett. 72, 2265 (1998)

${ }^{8}$ H. Lee, R. Lowe-Webb, W. Yang, and P. C. Sercel, Appl. Phys. Lett. 72, 812 (1998).

${ }^{9}$ B. A. Joyce, T. S. Jones, and J. G. Belk, J. Vac. Sci. Technol. B 16, 2373 (1998).

${ }^{10}$ P. Geng, J. Márquez, L. Geelhaar, J. Platen, C. Setzer, and K. Jacobi, Rev. Sci. Instrum. 71, 504 (2000).

${ }^{11}$ G. S. Solomon, J. A. Trezza, and J. S. Harris, Jr., Appl. Phys. Lett. 66, 3161 (1995); P. B. Joyce, T. J. Krzyzewski, G. R. Bell, T. S. Jones, S. Malik, D. Childs, and R. Murray, Phys. Rev. B 62, 10891 (2000).

${ }^{12}$ L. Geelhaar, J. Márquez, and K. Jacobi, Phys. Rev. B 62, 6908 (2000).

${ }^{13}$ L. Geelhaar, J. Márquez, P. Kratzer, and K. Jacobi, Phys. Rev. Lett (to be published)

${ }^{14}$ S. Guha, A. Madhukar, and K. C. Rajkumar, Appl. Phys. Lett. 57, 2110 (1990).

${ }^{15}$ M. D. Pashley, Phys. Rev. B 40, 10481 (1989). 